\title{
Three-Dimensional Myocardial Perfusion Maps by Contrast Echocardiography
}

\author{
ARIC A. AIAZIAN, M.D., DILA ATAOULLAKHANOVA, M.D., WIM VLETTER, M.Sc., \\ NATALIA VARCHENKO, M.S., KONSTANTIN GANKIN, M.S., \\ FOLKERT J. TEN CATE, M.D., PATRICK W. SERRUYS, M.D., \\ and JOS R.T.C. ROELANDT, M.D. \\ Thoraxcenter, Erasmus University, and University Hospital, Rotterdam, The Netherlands
}

We evaluated the clinical applicability of a system for three-dimensional (3-D) display of a perfusion map following myocardial contrast echocardiography (MCE). The system was used in 12 patients (9 males and 3 females, mean age $52 \pm 10$ years) undergoing interventional treatment of chronic total coronary occlusion. In each patient three standard apical views were acquired at baseline with sonicated Iopamidol ${ }^{n}$ injections into the left coronary artery (LCA) and into the right coronary artery (RCA). Following successful recanalization of the occluded artery MCE was repeated. The patients tolerated the procedure well. Acquisition of three standard apical views provided sufficient information for the reconstruction of 3-D perfusion maps containing the 16 standard left ventricular $(L V)$ segments. Side-by-side display of the perfusion maps obtained following LCA and RCA echocontrast injections allowed us to classify the myocardial segments (192) into three groups: (1) those supplied by one major artery (124); (2) those supplied by collaterals from contralateral or both major arteries (58); and (3) segments supplied by none of the major arteries (10). Decreased opacification was observed in 50 segments of group 2. Following successful intervention we were able to visualize the redistribution of blood flow delivered to the LV myocardium by each major coronary artery in 3-D format. We conclude that this 3-D approach, which can easily be performed with currently available ultrasound equipment, allows an estimate of the contribution of each major coronary artery to $L V$ perfusion before and after coronary angioplasty. (ECHOCARDIOGRAPHY, Volume 14, July 1997)

three-dimensional echocardiography, myocardial contrast echocardiography, cardiac ultrasound

Myocardial contrast echocardiography (MCE) is a promising technique for assessment of myocardial perfusion in patients undergoing percutaneous transluminal coronary angloplasty ${ }^{1-3}$ or bypass surgery. ${ }^{4}$ However, the data provided by MCE are limited to one cross-sectional view of the left ventricle and do not allow quantitation of the myocardial blood flow in the clinical setting. It would be desirable to obtain images comparable to the three-dimensional (3-D) display of myocardial perfusion provided by nuclear imaging techniques such as single photon emission

Address for correspondence and reprints: J.R.T.C. Roelandt, M.D., Erasmus University, Rotterdam, Thoraxcenter Bd 406, Dr. Molewaterplein 40, 3015 GD Rotterdam, The Netherlands. Fax: 31-10-4363096. computed tomography (SPECT). Three-dimensional reconstruction using echocardiographic images obtained by a rotating ultrasound transducer has been used to visualize and quantitate the myocardial area at risk during MCE. However, as a result of the relatively long image acquisition time this approach requires injection of a deposit echocontrast agent, which stays in the myocardium for a long time. ${ }^{5}$ Currently such an agent is not yet available for clinical use. Furthermore, this approach is not suitable for comparative analysis of contrast opacification in different myocardial segments due to different time intervals between the contrast injection and acquisition of different myocardial planes.

To avoid these limitations we developed a modified MCE technique with acquisition of 
several left ventricular (LV) views during multiple echocontrast injections into the left coronary artery (LCA) and the right coronary artery (RCA), which allowed 3-D reconstruction of myocardial perfusion map using standard apical views obtained by transthoracic echocardiography. ${ }^{6,7}$ In this article, we describe our initial clinical experience with this technique and equipment.

\section{Methods}

\section{Study Patients}

We studied 12 patients (9 males and $3 \mathrm{fe}$ males, mean age $52 \pm 10$ years) with chronic total occlusion of one of the major coronary arteries (RCA) in ten patients and left anterior descending artery (LAD) in two patients. Exclusion criteria were significant stenosis of other than the occluded coronary artery and technically poor quality of the echocardiogram. Patients were selected from a population enrolled in a large study at our institution designed to determine the feasibility of the treatment of chronically occluded coronary artery by laser angioplasty. All the patients gave informed consent. Myocardial contrast echocardiography was performed after the diagnostic coronary angiography and just before laser angioplasty.

The echocontrast agent (Sonicated Iopamidol ${ }^{\mathrm{R}}$ [Bracco, Milan, Italy]) prepared by a previously described method ${ }^{8}$ was injected into the coronary arteries. Two-dimensional images were obtained by a commercially available system (Sonos 1500, Hewlett-Packard, Andover, MA, USA) with a $2.5-\mathrm{MHz}$ transducer and recorded on $1.25-\mathrm{cm}$ VHS video tape. The acquisition of the images was started before the echocontrast injection (baseline recording). Three apical views of the left ventricle (four-chamber, longaxis, and two-chamber) were subsequently obtained. Each view was recorded in real time for about 1 minute. In the same way, these views were obtained with the injection of $5 \mathrm{~mL}$ of echocontrast into the RCA in each view. The procedure was repeated with the echocontrast injections into the LCA. Imaging was continued until complete washout of the contrast. Image acquisition and injection were then repeated in the next view. Thus, a total of six injections of echocontrast were performed. The entire procedure took approximately 6 minutes.

Coronary angiography, and the same order and number of myocardial contrast injections, were repeated immmediately after the interventional procedure.

\section{Analysis}

We developed a semiquantitative approach similar to the one used in nuclear cardiology for analysis. The image analysis and reconstruction of 3-D perfusion maps was performed by a dedicated workstation for contrast echocardiography (Axle, The Netherlands). The end-diastolic frames from the video recordings were digitized and analyzed semiautomatically. The LV myocardium was divided into 16 segments and the intensity in each segment was automatically measured and displayed in arbitrary intensity units (IUs). In each segment changes in the intensity were plotted as the time-intensity curve and automatically measured. The peak intensities automatically measured by the system showed an excellent correlation $(r=0.99)$ with the manual measurements of peak intensities from the same time-intensity curves (Fig. 1).

The quantitative data are stored as a dataset with quantitative parameters of each myocardial segment. For rapid visual comparison the peak contrast intensity of different segments was automatically encoded either in shades of gray or color coded. A relative color bar was used: dark blue for myocardial regions with the lowest intensity increase and white for the regions with the maximal intensity increase. Using this dataset a 3-D perfusion map of the left ventricle was constructed and displayed (Fig. 2 ). The color scheme (or gray scale) in each patient is the same, however, the absolute numbers are different. The minimal intensity increase in all the instances is displayed as dark blue (or black), but the actual measured contrast opacification in the intensity units is different in each patient as shown by the numbers along the bars (Figs. 3 and 4 ). The relative intensity units allow comparison of different regions with different intensities. Values below 5 IUs are not considered to be caused by 


\section{Maximal intensity increase}

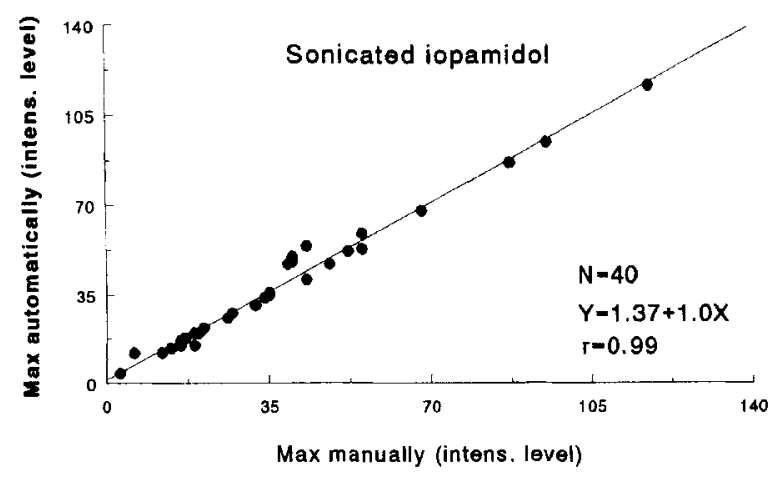

Figure 1. There was an excellent correlation between the measurements of maximal contrast intensity increase (in relative intensity level units) $o b$ tained manually (by manually driven cursor) and automatically from the same time-intensity curves with the intracoronary injections of sonicated Iopamidol.

the contrast injections, but reflect small fluctuations in the background intensity. Peak intensities of the different groups of segments were compared by the Student's $t$-test.

\section{Results and Illustrative Cases}

A 16-segment 3-D color-coded perfusion map of the LCA and RCA injections in each patient were reconstructed by the workstation and displayed side by side for comparative assessment. A representative example of LV 3-D perfusion maps in a patient with chronic LAD occlusion are shown in Figures $3 a$ and $3 b$.

Figure $3 \mathrm{a}$ was obtained after the LCA MCE injection. The anterior and anterolateral walls were not perfused, but the posterolateral walls (segment 5, 9, and 10) are perfused by the LCA and, partially, by the left circumflex artery. Perfusion of the anterior and anterolateral walls was visualized after the RCA injection. Thus, perfusion of the LAD territory is completely performed by the RCA in this patient with an LAD occlusion.

Dark blue areas show the segments with lower opacification, while the bright pink, yellow, and white segments represent the areas with a higher opacification level. In this patient the RCA supplies a significant part of the left ventricle, providing collaterals to the anterior and lateral free walls and the interventricular septum (Fig. 3b). The apical segments were not opacified by neither the LCA nor the RCA. These findings suggested that the apical part of the left ventricle is not viable.

Figures 3c and 3d show 3-D perfusion maps obtained in another patient with chronic RCA occlusion. The reconstructed image following RCA echocontrast injection (Fig. 3d) shows minimal intensity increase due to the fluctuations in the baseline intensity of the ultrasound image without any significant myocardial opacification (see also analysis paragraph). However, the LCA echocontrast injection (Fig. 3c) completely opacified the LV myocardium; the highest intensity increase was in the anterior wall and interventricular septum and the lowest was in the posterior and inferior walls supplied by collaterals from the LCA.

Such a side-by-side display of RCA and LCA injections allowed classification of all the myocardial segments in 12 patients (totally 192 supplied by segments) into three groups supplied by: (1) one major artery (124); (2) segments supplied by collaterals from contralateral or both major arteries (58); and (3) segments not supplied by one of the major coronary arteries and were considered as nonviable segments (10). Following the echocontrast injection the intensity increase measured in group 1 of the segments supplied by a normal coronary artery was $45.4 \pm 13.5$ relative IUs, while in group 2 of the collateralized segments subtended by an occluded coronary artery it was only $31.4 \pm 14.8 \mathrm{IU}$. The collateralized segments presented on a 3-D color-coded map as areas with decreased perfusion if compared with the segments supplied by a normal coronary artery $(\mathrm{P}<0.001)$.

Reconstruction of the LCA and the RCA perfusion maps by 3-D MCE allowed an estimation of the contribution of each major coronary artery to the perfusion of the LV myocardium and assessment of the source and level of perfusion of each of $16 \mathrm{LV}$ segments.

Myocardial contrast echocardiography and reconstruction of $3-D$ perfusion maps were repeated after the recanalization procedure. Figures $4 \mathrm{a}$ and $4 \mathrm{~b}$ represent $3-\mathrm{D}$ perfusion images 


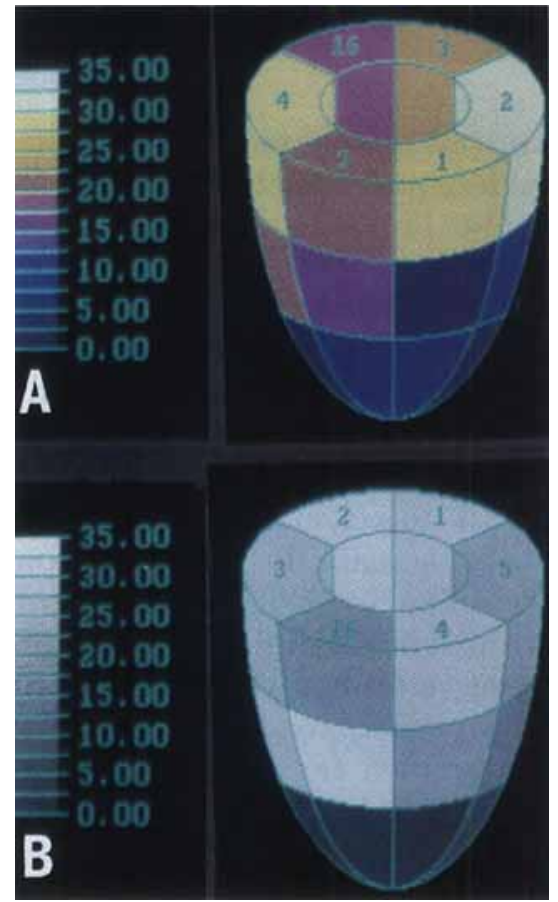

Figure 2. The color coding (A) or gray scale coding $(B)$ of myocardium is performed according to the relative bar. The lowest parameter value (intensity increase here) is dark blue or black, the highest is yellow or white.

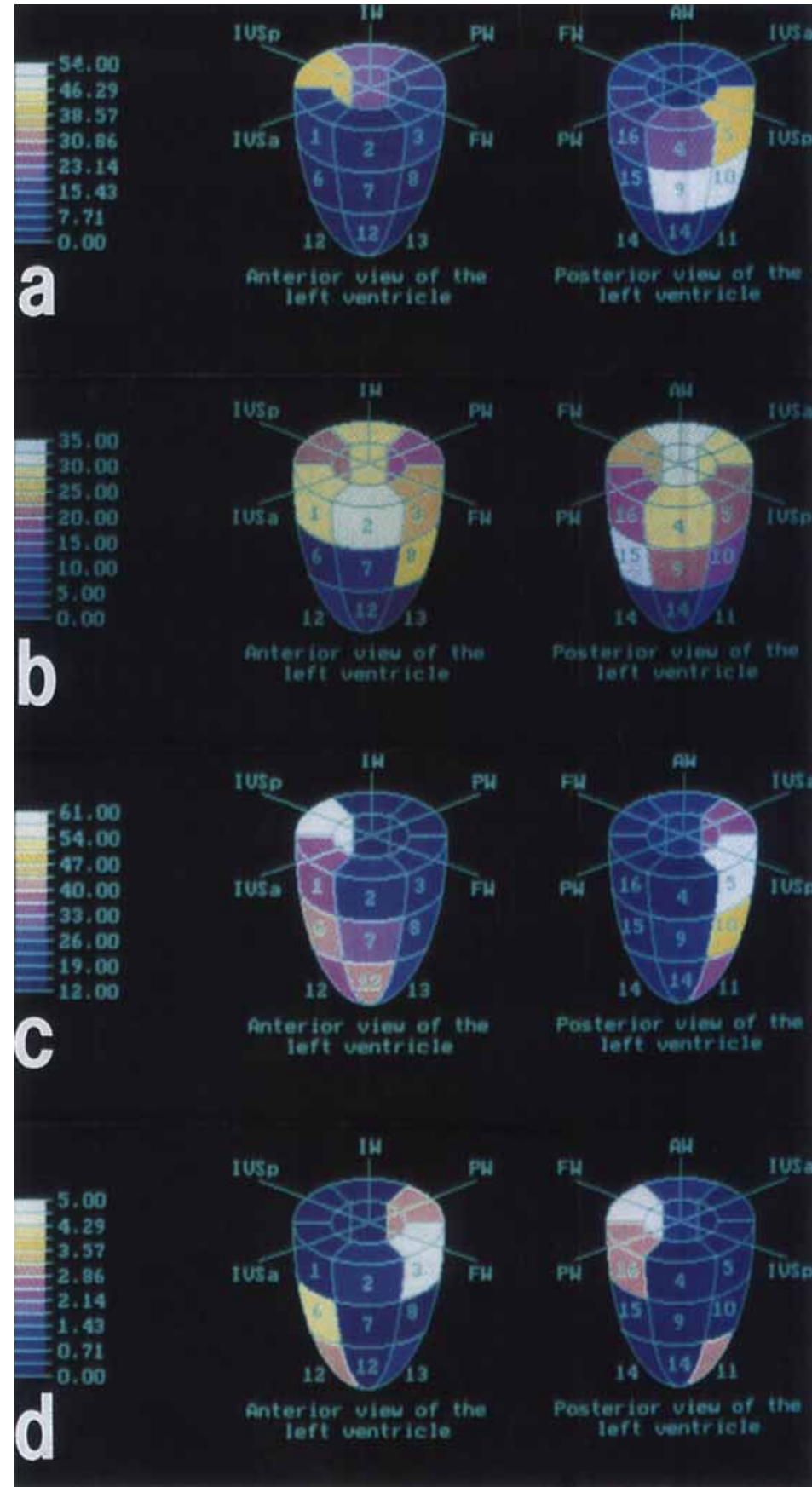

Figure 3. Three-dimensional (3-D) perfusion maps of the left ventricle in the patients with totally occluded left anterior descending $(L A D)(a, b)$ and right coronary artery $(R C A)(c, d)$. Anterior and posterior views of the left ventricle are displayed together following the same echocontrast injection. The images were obtained with echocontrast injections into the left coronary artery $(L C A)(a, c)$ and the RCA $(b, d) . A W=$ anterior wall of the left ventricle; $F W=$ free wall of the left ventricle; IVSa $=$ anterior part of the interventricular septum; IVSp = posterior part of the interventricular septum; $P W=$ posterior wall of the left ventricle. The numbers indicate the location of the respective standard myocardial segments. (a) LCA injection in the patient with $L A D$ occlusion a perfusion defect could be observed in the anterior wall, anterior part of interventricular septum, and apex. (b) RCA injection in the same patient with LAD occlusion shows that part of the anterior wall, free wall, and the anterior part of interventricular septum are supplied by collaterals from the RCA. Side-by-side assessment of the LCA (a) and $R C A(b)$ injection allowed to observance of the absence of opacification by either artery in the apex, which was not viable. (c) In the patient with RCA occlusion, LCA injection opacifies the entire left ventricle. Low intensity increase is observed in the inferior and posterior walls, which are supplied by a relatively poor collateral flow. Contrast opacification of the anterior wall and the anterior part of interventricular septum is bright and normal. (d) RCA injection in the same patient with RCA occlusion shows only minimal intensity increase (up to 5 IUs according to the color bar), which reflect the small fluctuations in the baseline intensity of the ultrasound image without any significant myocardial opacification 


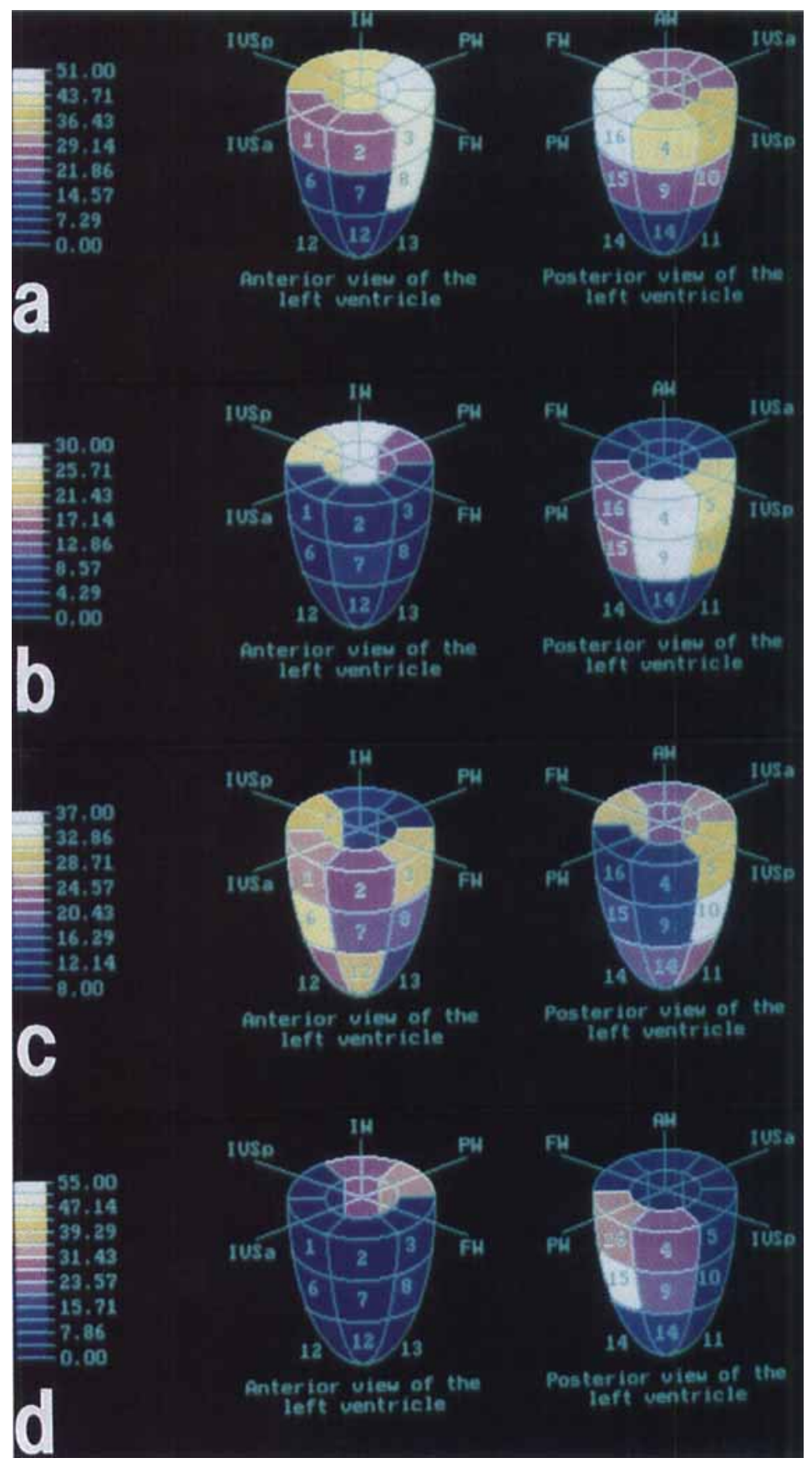

Figure 4. Three-dimensional (3-D) perfusion maps obtained following successful opening of the left anterior descending artery $(L A D)(a, b)$ and right coronary artery $(R C A)(c, d)$ occlusions. The echocontrast injections were performed into the left coronary artery $(L C A)(a, c)$ and into the $R C A(b, d)$. (a) Following the successful opening of the LAD, LCA supplied the anterior wall, free wall, and the anterior part of interventricular septum (compare with Fig. 3 a obtained in the same patient before the intervention). (b) Three-dimensional image of the RCA injection in the same patient shows amelioration of collateral flow from the RCA to the anterior wall, and the anterior part of interventricular septum (compare with Fig. $3 b$ ). (c) In a patient with successfully treated RCA occlusion LCA injection shows significantly decreased collateral flow to the posterior and the inferior walls (compare with Fig. 3c). (d) In the same patient the RCA after the intervention supplies the inferior and the posterior walls. The established antegrade flow provides bright opacification (compare with Fig. $3 d$ ). 
after recanalization of the LAD occlusion (compare with the images obtained in the same patient before the intervention, Figs. $3 a$ and $3 b$ ). RCA injection showed amelioration of collateral flow to the anterior wall and the anterior part of the interventricular septum.

Figures $4 \mathrm{c}$ and $4 \mathrm{~d}$ show representative examples of 3-D LV perfusion maps in a patient following a successful recanalization of RCA occlusion (compared with the 3-D perfusion maps reconstructed before the treatment, Fig. 3d). Collateral flow from the LCA to the posterior and inferior walls significantly decreased. The antegrade flow delivered to these walls by the RCA provides bright opacification. In the ten patients with RCA occlusion $2 \%$ of the LV segments changed their supply from LCA to RCA after successful opening of the RCA, and $17 \%$ of segments were still perfused from the RCA while collateral supply from the LCA still was seen.

In the patients with $\mathrm{LAD}$ occlusion, $25 \%$ of all the LV segments changed their supply from RCA to LCA and 18\% received the flow both from LCA and RCA after the recanalization procedure. Thus, 3-D perfusion maps allowed observation of the redistribution of myocardial perfusion following the interventional treatment.

After the successful recanalization of the occluded artery the peak intensity in the segments, which obtained the blood from the opened artery, showed mean increase by $7.3 \mathrm{IUs}$ compared to the opacification of these segments before the interventional treatment $(\mathrm{P}<0.05)$.

\section{Discussion}

Three-dimensional perfusion mapping by MCE has potential for assessment of myocardial viability and ischemia since the presence, site, and extent of myocardial perfusion abnormalities can be evaluated.

Previous studies have shown that myocardial viability can be predicted by defining the areas of preserved microvascular integrity as assessed by MCE. ${ }^{9,10}$ The method as described in the present study allows detection of segments, which do not opacify following contrast injection into the LCA or the RCA. These findings therefore suggest that these segments have no detectable microvascular perfusion.
We utilized a minimal number of $L V$ views (three standard apical views) including the 16 standard LV segments as comparable to analysis done for stress echocardiography. ${ }^{11}$ More views would allow reconstruction of $3-D$ perfusion maps with more precise delineation of myocardial segments but at significant prolonged acquisition times.

The dataset of 3-D perfusion maps in the Axle workstation contains complete information about the contrast washin and washout in each myocardial segment. Due to full processing of the time-intensity curves the peak and time of peak intensity are always correctly identified, as are the other relevant quantitative parameters such as area under the time-intensity curve, contrast washout half-time, etc. The results of this complex analysis are automatically displayed as a set of 3-D maps representing relative perfusion of each myocardial segment.

The voxel or volume recording technique provides a different presentation of 3-D perfusion based on a number of sequential $L V$ views acquired by a rotating ultrasound transducer. ${ }^{5}$ Due to the fact that each of the views is obtained by the transducer at different time intervals after the echocontrast injection this technique cannot be utilized to study the timing of peak intensity. This limitation holds true also for the other parameters of the time intensity curves. Three-dimensional MCE is a feasible and attractive alternative method for perfusion imaging during cardiac catheterization.

The other perfusion imaging techniques such as thallium-201 imaging (SPECT) and position emission tomography (PET) use nuclear cameras, which lack the versatility of ultrasound machines. ${ }^{12-14}$ In addition, thallium scans sometimes have to be repeated several times. However, PET gives specific information about myocardial flow and metabolism but at an increased cost. Also PET is not widely available.

\section{Current Limitations of the Method}

If the backscatter ultrasound signals are displayed in intensities these signals are significantly influenced by electronic signal processing. ${ }^{15}$ Attenuation and poor lateral resolution in the original ultrasound images may signifi- 
cantly influence analysis. On the other hand, hyperemia ${ }^{16}$ may also increase the backscatter ultrasound intensities.

Thus, equipment characteristics and biological factors may influence MCE images. These limitations must be taken into account for the analysis.

\section{Conclusion}

Current advances in image analysis technology suggest that 3-D myocardial perfusion mapping with contrast echocardiography may be applied clinically. The instrumentation and technique utilized in this study can be readily used in a clinical setting during cardiac catheterization and interventional procedures.

Comparative assessment of 3-D myocardial perfusion maps obtained in patients after LCA and RCA echocontrast injections allows an estimation of the relative contribution of each major coronary artery to $L V$ myocardial perfusion. In the present study MCE in 3-D also allowed observation of the redistribution of myocardial perfusion following a successful intervention. Further studies are required to validate the results of 3-D myocardial perfusion mapping with contrast echocardiography and to determine its clinical usefulness.

\section{References}

1. Lang R, Feinstein S, Feldman T, et al: Contrast echocardiography for evaluation of myocardial perfusion: Effects of coronary angioplasty. J Am Coll Cardiol 1986;8:323-335.

2. Reisner S, Ong L, Lichtenberg G, et al: Quantitative assessment of the immediate results of coronary angioplasty by myocardial contrast echocardiography. $J$ Am Coll Cardiol 1989;2: 52-856.

3. Porter TR, D'Sa A, Pesko L, et al: Usefulness of myocardial contrast echocardiography in detecting the immediate changes in anterograde blood flow reserve after coronary angioplasty. Am J Cardiol 1993;71:893-896.

4. Spotnitz WD, Kaul S: Intraoperative assessment of myocardial perfusion using contrast echocardiography. Echocardiography 1990;7:209-227.

5. Kasprzak JD, Ten Cate FJ, Sutton A, et al: Dynamic three dimensional myocardial perfusion echocardiography using a deposit contrast agent. Circulation 1995; 92(suppl):I-261.

6. Ten Cate FJ, Aiazian AA, Hamburger J, et al: Reconstruction of three-dimensional perfusion maps in humans by myocardial contrast echocardiography. J Am Coll Cardiol 1995; Feb (suppl):247A.

7. Ten Cate FJ, Aiazian AA, de Jong N. Advances in clinical contrast echocardiography: The Thoraxcentre experience. Thoraxcentre $J$ 1995;7/4: 13-14.

8. Ten Cate FJ, Serruys PW, Huang H, et al: Is the rate of disappearance of echo contrast from the interventricular septum a measure of left anterior descending coronary artery stenosis? Eur Heart $J$ 1988;9:728-733.

9. Ragosta M, Camarano G, Kaul S, et al: Microvascular integrity indicates myocellular viability in patients with recent myocardial infarction. New insights using myocardial contrast echocardiography. Circulation 1994;89: 2562-2569.

10. Vandenberg BF, Kerber RE, Skorton DJ. Detection of myocardial viability with ultrasound tissue characterization: Myocardial contrast echocardiography and integrated backscatter imaging. Am $J$ Card Imaging 1994;8:113-122.

11. Bourdillon PDV, Broderick TM, Sawada SG, et al: Regional wall motion index for infarct and non-infarct regions after reperfusion in acute myocardial infarction: Comparison with global wall motion index. I Am Soc Echocardiogr 1989;2:398-407.

12. Miller DD, Verani MS: Current status of myocardial perfusion imaging after percutaneous transluminal coronary angioplasty. $J \mathrm{Am}$ Coll Cardiol 1994;24:260-266.

13. Garvin AA, Cullom SJ, Garcia EV: Myocardial perfusion imaging using single-photon emission computed tomography. Am J Card Imaging 1994;8:189-198.

14. Sinha S, Sinha U, Czernin J, et al: Noninvasive assessment of myocardial perfusion and metabolism: Feasibility of registering gated MR and PET images. Am J Roentgenol 1995;164:301-307.

15. Zwehl W, Areeda J, Schwartz G, et al: Physical factors influencing quantitation of two-dimensional contrast-echo amplitudes. J Am Coll Cardiol 1984;4:157-164.

16. Kondo S, Tei C, Meerbaum S, et al: Hyperemic response of intracoronary contrast agents during two-dimensional echographic delineation of regional myocardium. J Am Coll Cardiol 1984; 4:149-156. 